\title{
Curativo de celulose bacteriana para o tratamento de lesões por pressão em pacientes hospitalizados
}

\section{Dressing of bacterial cellulose for the treatment of pressure injuries in hospitalized patients}

\author{
Glícia Maria de Oliveira' • Janaína Maria Silva Vieira² • Jaiurte Gomes Martins da Silva ${ }^{3}$ \\ Érica Larissa Marinho Souto de Albuquerque ${ }^{4} \bullet$ Amanda Vasconcelos de Albuquerque $^{5}$ \\ José Lamartine de Andrade Aguiar ${ }^{6}$ Flávia Cristina Morone Pinto ${ }^{7}$
}

\begin{abstract}
RESUMO
Objetivou-se monitorar a evolução do processo cicatricial das lesões por pressão (LP) em pacientes hospitalizados em Unidades de Terapia Intensiva (UTI), a partir da aplicação da cobertura de celulose bacteriana (CB). Trata-se de uma série de casos, amostra constituída por 10 pacientes internados na UTI. Foram excluídos crianças, adolescentes, e pacientes com outros tipos de lesões de pele. Foram aplicadas as escalas preditivas de Braden e a de MEASURE. Realizado monitoramento fotográfico pelo programa MOWA®.A pesquisa foi desenvolvida no setor de terapia intensiva de um hospital univer-

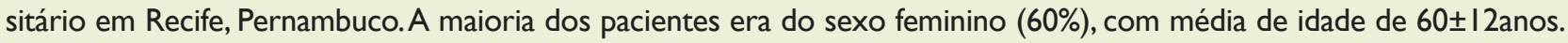
Quanto as comorbidades, 3 pacientes apresentavam diabetes mellitus (DM), 2 hipertensão arterial sistêmica (HAS), 3 DM e HAS e 2 negaram doença crônica. Sete pacientes foram acompanhados por 30 dias, em média. $O$ risco avaliado pela escala de Braden foi de 9,66. As LP localizavam-se na região sacral, com área média de $84,48 \mathrm{~cm} 2$ à avaliação inicial. Após 30 dias de acompanhamento observou-se redução da área média das $L P\left(-14,7 \mathrm{~cm}^{2}\right)$. A CB funcionou como barreira física e indutor do remodelamento tecidual.
\end{abstract}

Palavras -chave: Cicatrização; Lesão por pressão; Celulose; Cana-de-açúcar.

\begin{abstract}
The objective of this study was to monitor the evolution of the cicatricial process of pressure lesions (LP) in patients hospitalized in Intensive Care Units (ICU), from the application of bacterial cellulose (CB) coverage. This is a series of cases, a sample consisting of 10 patients hospitalized in the ICU. Children, adolescents, and patients with other types of skin lesions were excluded. Predictive scales of Braden and MEASURE were applied. Photographic monitoring carried out by the MOWA® program. The research was developed in the intensive care sector of a university hospital in Recife, Pernambuco. The majority of the patients were female (60\%), with a mean age of $60 \pm 12$ years. As for comorbidities, 3 patients had diabetes mellitus (DM), 2 systemic arterial hypertension (SAH), 3 DM and SAH and 2 denied chronic disease. Seven patients were followed for 30 days, on average. The risk assessed by the Braden scale was 9.66. LPs were located in the sacral region, with a mean area of $84.48 \mathrm{~cm} 2$ at the initial evaluation. After 30 days of follow-up, we observed a reduction in the mean area of LP $\left(-14.7 \mathrm{~cm}^{2}\right)$. CB worked as a physical barrier and inducer of tissue remodeling.
\end{abstract}

Keywords: Wound healing; Pressure injury; Cellulose; Sugar cane.

'Acadêmica de Enfermagem, Núcleo de Enfermagem, Centro Acadêmico de Vitória, Universidade Federal de Pernambuco,Vitória de Santo Antão, PE, Brasil. glliciaoliveira@gmail.com (Trabalho de conclusão de curso de graduação);

2Enfermeira, Unidade de Terapia Intensiva, Hospital das Clínicas, Universidade Federal de

Pernambuco, Recife, PE, Brasil. j.janaínavieira@hotmail.com

${ }^{3}$ Biólogo, Docente pelo Departamento de Anatomia, Centro de Biociências, Universidade Federal de Pernambuco, Recife, PE, Brasil.jaiurte@hotmail.com

${ }^{4}$ Enfermeira, Mestre, Unidade de Gerenciamento de Riscos Assistenciais, Hospital das Clínicas, Universidade Federal de Pernambuco, Recife, PE, Brasil. ericalarissaalbuquerque@gmail.com

${ }^{5}$ Bióloga, Núcleo de Cirurgia Experimental, Centro de Ciências da Saúde, Universidade Federal de Pernambuco, Recife, PE, Brasil. amandabiocel@gmail.com 6Médico, Livre-docente, Núcleo de Cirurgia Experimental, Departamento de Cirurgia, Universidade Federal de Pernambuco, Recife, PE, Brasil. aguiarjl@terra. com.br

${ }^{7}$ Enfermeira, Doutora, Docente pelo Núcleo de Nutrição, Centro Acadêmico de Vitória, Universidade Federal de Pernambuco, Vitória de Santo Antão, PE, Brasil. fcmorone@gmail.com 


\section{INTRODUÇÃO}

Lesão por pressão (LP) é um dano identificado na pele e/ou tecidos moles subjacentes, geralmente sobre uma proeminência óssea ou referente ao uso de dispositivo médico ou a outro artefato.A LP pode se manifestar em pele íntegra ou como úlcera aberta e pode ser pungente'. A origem da LP é multifatorial e provém de fatores de risco extrínsecos e intrínsecos ${ }^{2}$. A resistência do tecido mole à pressão e ao cisalhamento pode também ser prejudicada pelo microclima, nutrição, perfusão, comorbidades e pela sua condição'.

A constrição do tecido sensível sobre a proeminência óssea resulta subsequentemente na diminuição do fluxo sanguíneo e isquemia do local, causando hipóxia dérmica, necrose, ruptura da epiderme e eventualmente contaminação bacteriana ${ }^{2}$.

A ocorrência da LP é comum em pessoas idosas e em pacientes acometidos por doenças crônico-degenerativas. Tal agravo gera um aumento de custo à instituição, devido ao aumento no tempo de internação e aos custos associados ao tratamento, além de aumentar a carga de trabalho da equipe de saúde ${ }^{3}$.

Os clientes de Unidades de Terapia Intensiva (UTI) são os mais desfavorecidos quanto a conservar a pele íntegra desde o primeiro dia de UTI, possuindo alto risco, principalmente pela restrição de atividade física e mobilidadade ${ }^{4}$.

A produção e investigação de novos produtos biomédicos são de grande relevância para o desenvolvimento de novos tratamentos de saúde e para o aperfeiçoamento da qualidade de vida ${ }^{5}$. Neste sentido, a membrana de celulose bacteriana, proveniente do melaço de cana de açúcar, produzida na Estação Experimental de Cana de Açúcar em Carpina (EECC), da Universidade Federal Rural de Pernambuco (EECA/UFRPE) uma alternativa promissora no tratamento de feridas.

Um estudo recente sobre os efeitos de citotoxicidade aguda, genotoxicidade e antigenotoxicidade da CB, preparados nos testes in vitro e in vivo em ratos wistar machos e fêmeas, apontou que o biopolímero da cana de açúcar testado não foi citotóxico e nem genotóxico, caracterizando a $\mathrm{CB}$ como um produto biocompatível e atóxico ${ }^{7}$. Assim, além do seu potencial terapêutico, a CB contém atributos em termos de segurança ${ }^{7,8}$.

Um estudo experimental da citotoxicidade da CB foi examinado in vitro frente a dois outros biomateriais: polipropileno e e-PTFE. A CB manifestou baixa citotoxicidade comparável ao e-PTFE por meio do índice de adesão, produção de óxido nítrico e a viabilidade celular de macrófagos alveolares em ratos ${ }^{8}$.

Analises de biocompatibilidade da CB como agente de preenchimento na bexiga de coelhos, expressou menor resposta inflamatória e se integrou melhor ao tecido hospedeiro do que o grupo tratado com dextranômero (Deflux®) ${ }^{9}$.
O gel de CB também foi testado no tecido subcutâneo dos coelhos, buscando analisar a reação de sensibilidade local e a biocompatibilidade. Os autores constataram que houve formação de neovasos em $84,4 \%$ na área de implante do gel, intensidade de infiltrado inflamatório estatisticamente considerável e fibrogênese de grau I com maior prevalência nos grupos CB. Corroborando a biocompatibilidade, a indução tecidual e a integração da $\mathrm{CB}$ no tecido subcutâneo de coelhos ${ }^{10}$.

Em um estudo pioneiro com uso da membrana da $C B$ no tratamento de feridas de membros inferiores resultantes de doenças vasculares em seres humanos, pode-se observar que no grupo $\mathrm{CB}$, em mais de $80 \%$ dos pacientes (versus $60 \%$ no grupo de controle) as úlceras eram mais superficiais no final do tempo de observação'.

As LP causam dor e sofrimento ao portador como também aumentam o tempo de internação do paciente, trata-se de uma problemática dispendiosa, vivenciada corriqueiramente no âmbito hospitalar. A produção de novos produtos é de fundamental importância, principalmente síntese dos biomateriais, que viabilizam redução nos custos nesta terapêutica. O biopolímero celulósico é uma cobertura, indutora do processo cicatricial, de baixo custo e de fonte renovável.

\section{MÉTODO}

\section{Desenho do Estudo}

Trata-se de uma série de casos (I0) alinhados metodologicamente à pesquisa do tipo experimental, inserida na linha de estudos com biopolímeros de cana de açúcar. Considera-se que os estudos que abrangem os indivíduos são os relatos de caso, relatos de séries de casos, estudos transversais e observacionais".

A amostragem foi por conveniência, constituída a partir da seleção de 10 pacientes internados em regime de longa permanência no HC/UFPE, de acordo com os critérios de inclusão e exclusão definidos previamente. A amostragem por conveniência é moldada e frequentemente empregada para geração de ideias em pesquisas exploratórias, principalmente ${ }^{12}$.

Foram incluídos pacientes adultos com LP, hospitalizados no HC/UFPE no momento da coleta de dados para pesquisa e que se enquadrassem nos graus de lesão tissular, conforme definido pela National Pressure Ulcer Advisory Panel (2016) independentemente do número de úlceras ou localização, ainda que existisse a necessidade de debridamento para remoção do tecido necrótico ou desvitalizado, independente do diagnóstico principal vinculado à internação'.

Foram excluídos do estudo crianças e adolescentes. Pacientes adultos com outros tipos de úlceras/lesões de pele que não sejam especialmente as por pressão também foram excluídos da pesquisa. 
Todos os pacientes recrutados, após esclarecimentos sobre a pesquisa e aprovação dos familiares, já que os mesmos não tinham condições mentais para responder, receberam o curativo de $\mathrm{CB}$ e foram acompanhados desde a inclusão na pesquisa até a alta hospitalar ou óbito.

Esta pesquisa foi aprovada pelo Comitê de Ética em Pesquisa com seres humanos (CEP), da Universidade Federal de Pernambuco ( $\mathrm{N}^{\circ}$. I.II4.716) e na Plataforma Brasil CAAE ( $N^{\circ}$. 330993 I4.9.0000.5208), obedecendo aos princípios Éticos da Pesquisa com Seres Humanos determinados pelo Conselho Nacional de Saúde na resolução 466/20I2 (CNS, 20I2) e consoante à Declaração de Helsinki revisada em 2000.

\section{O curativo}

O biopolímero celulósico é um exopolissacarídeo, obtido a partir do melaço de cana açúcar, produzido na estação experimental de Carpina, unidade pertencente a Universidade Federal Rural de Pernambuco (UFRPE). Os curativos de CB foram fornecidos pela POLISA Biopolímeros para a Saúde Ltda.

Foram utilizadas membranas perfuradas acopladas a uma esponja, ambas de biopolímero, na forma de filmes estéreis, nas dimensões de $10 \times 8 \mathrm{~cm}$ e 0,01 a $0,02 \mathrm{~mm}$ na espessura, embalados em envelopes grau cirúrgico, separadamente.

A CB expressa baixa citotoxidade, alta biocompatibilidade, elasticidade, flexibilidade, resistência a tração, e ainda pode modelado em várias formas, preenchendo assim as exigências necessárias para as manufaturas dos implantes biológicos ${ }^{8}$.

\section{Procedimentos Clínicos}

Foram estabelecidas etapas dos cuidados de enfermagem orientados pela Sistematização da Assistência de Enfermagem (SAE) que num sentido geral correspondem à identificação do problema (coleta de dados e diagnósticos) seguindo planejamento das intervenções, implementação dos cuidados de enfermagem e finalmente, a reavaliação do plano de cuidados.

\section{Coleta de dados e diagnósticos}

$\mathrm{Na}$ etapa inicial, foram utilizados dados primários (anamnese, exame físico, aplicação de escalas de Braden e MEASURE) e dados secundários (prontuários, fluxograma de exames, familiares). A partir dos dados coletados foi realizada uma análise do(s) problema(s), sua etiologia, sinais e sintomas (Formulário de coleta de dados).

\section{Planejamento das intervenções}

O plano de cuidados foi elaborado com base nas prioridades identificadas, para os pacientes críticos a prioridade é fisiológica, visando o reestabelecimento da mesma. Porém neste momento podem-se incluir as dimensões sociais e psicológicas, além da lesão cutânea. Por seguinte, o estabelecimento dos resultados esperados, como: diminuição dos riscos, redução da dor e indução do processo cicatricial.

\section{Implementação dos cuidados}

Baseada em princípios científicos e na individualização do sujeito, realizado de acordo com as necessidades do paciente, após a avaliação clínica foi realizado o curativo, de acordo com os Procedimentos Operacionais Padronizados na Unidade de Terapia Intensiva, em conformidade com orientações do Serviço de Controle de Infecção Hospitalar, do HC/UFPE, sucedendo as seguintes etapas: Desbridamento: Realizado para remoção do tecido necrótico ou desvitalizado, a fim de promover uma aceleração dos processos de reparação e regeneração tecidual e viabilizando a aplicação da cobertura; Limpeza: Solução salina (SF 0,9\%) para realização da limpeza, de

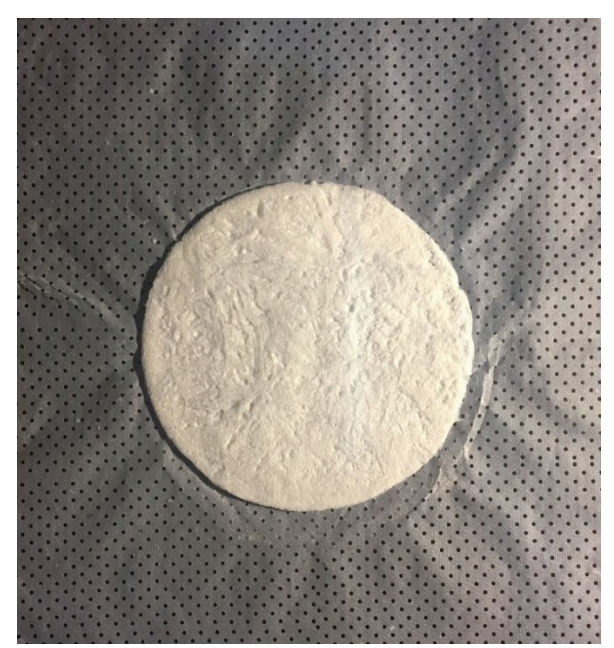

FIGURA 1 - Membrana de biopolímero celulósico 
modo a evitar traumatismo no leito da lesão, em jato de irrigação com pressão ideal, utilizando gazes umedecidas exercer pressão e friç̧ão suaves; Registro fotográfico: Foi realizado o registro fotográfico na avaliação inicial e nas reavaliações semanais para monitorização das respostas às medidas terapêuticas, com a câmera do apareIho celular MotoG5 plus; e, Cobertura: Após aplicação da cobertura de $C B$, foi realizado um curativo secundário com gaze, em alguns casos como barreira física foi utilizado o filme transparente a fim de impermeabilizar o local do curativo.

Foi avaliado como desfecho primário o processo de cicatrização (características teciduais) e como secundário o tempo de cicatrização.

\section{Reavaliação do plano de cuidados}

Durante as reavaliações semanais foram analisados os aspectos clínicos do paciente, a necessidade de interrupção do plano terapêutico, continuação ou modificação, conforme as necessidades apresentadas. As reavaliações aconteceram semanalmente com a aplicação de escalas preditivas, como a de Braden que avalia o risco para o desenvolvimento das LP e a MEASURE para avaliação clínica da ferida (Formulário de Reavaliação).

A escala de Braden está amparada na fisiopatologia das lesões por pressão e permite avaliação de aspectos importantes à formação da lesão, conforme seis parâmetros: percepção sensorial, umidade, mobilidade e atividade, nutrição, fricção e cisalhamento. Os cinco primeiros subescores recebem uma pontuação que varia de um a quatro, enquanto que o subescore fricção e cisalhamento, de um a três ${ }^{4}$.
O MEASURE resume as características da ferida que devem ser abordados na análise. As letras do acrônimo significam: M (measure - medida), E (exudate - exsudato), A (appearance - aparência), S (suffering - dor), U (undermining - descolamento), R (re-evaluation - reavaliação), e, E (edge - borda) ${ }^{13}$, (Tabela I).

\section{Procedimentos Estatísticos}

Realizada estatística descritiva, onde as frequências foram representadas em porcentagem e os dados contínuos foram apresentados como média e desvio padrão. Foram utilizadas tabelas para sumarizar os resultados.

\section{RESULTADOS}

Foram incluídos no estudo 10 participantes, $60 \%$ do sexo feminino e $40 \%$ masculino. $A$ idade variou entre 4 I a 75 anos, com média de idade de $60 \pm 12$ anos.

As medidas antropométricas foram mensuradas e o peso médio dos participantes foi de $68,2 \pm 11,16 \mathrm{~kg}$. A altura média foi de $1,61 \pm 0,09 \mathrm{~m}$. O Índice de Massa Corporal (IMC) médio foi de $26,67 \pm 5,3$ I valor referente a sobrepeso.

Quanto às comorbidades, 3 pacientes (30\%) apresentavam diabetes mellitus (DM), 2 hipertensão arterial sistêmica (HAS, 20\%), 3 DM e HAS associadas (30\%) e 2 não possuíam qualquer doença crônica (20\%).

Com relação aos medicamentos, metade dos pacientes $(50 \%)$ utilizava anti-hipertensivos, $40 \%$ faziam uso de hipoglicemiantes orais e $60 \%$ estavam em uso de insulinoterapia.

Os motivos de internação dos participantes do estudo estão descritos abaixo na Tabela 2.

Os níveis séricos de hematócrito $(\mathrm{Ht})$ e hemoglobina

TABELA 1 - Classificação MEASURE para avaliação clínica das feridas14

\begin{tabular}{l|l}
\hline Parâmetro & Conteúdo \\
\hline M Measure: Medida & Comprimento, largura, profundidade e área \\
\hline E Exsudate: Exudato & Quantidade e qualidade \\
\hline A Appearance: Aparência & Leito da ferida, tipo e quantidade de tecido \\
\hline S Suffering: Dor & Tipo e intensidade de dor \\
\hline U Undermining: Descolamento & Presença ou ausência \\
\hline R Re-evaluation: Reavaliação & Monitoração periódica de todos os parâmetros \\
\hline E Edge: Borda & Condição das bordas e do tecido adjacente \\
\hline
\end{tabular}

Fonte: Crozeta ${ }^{14}$

TABELA 2 - Descrição dos motivos de internação dos participantes.

\begin{tabular}{l|c}
\hline Diagnóstico(s) Médico(s) Principal(is) & Número de pacientes \\
\hline Insuficiência Cardíaca (IC) descompensada & 1 \\
Endocardite Infecciosa & 1 \\
Rebaixamento do Nível de Consciência (RNC) secundário ao Acidente Vascular Cerebral & 1 \\
Ureterolitíase & 1 \\
Síndrome Cardiorrenal & 1 \\
IC, RNC e Vasculopatia & 1 \\
RNC secundário à Encefalopatia Hepática & 1 \\
Câncer de Mama & 1 \\
Adenocarcinoma de próstata & 1 \\
Linfoma de Hodking e Lesão Cerebelar & 1 \\
\end{tabular}


$(\mathrm{Hb})$ foram registrados e apresentaram valor global médio de $23,91 \pm 0,97 \%$ e $8,76 \pm 2,39 \mathrm{~g} / \mathrm{dL}$, respectivamente. $O$ valor médio de $\mathrm{Ht}$ em mulheres foi $24,02 \%$ e para o sexo masculino foi de $23,91 \%$. Os níveis de $\mathrm{Hb}$ apresentaram valor médio de $8,56 \mathrm{~g} / \mathrm{dL}$ para mulheres e $8,90 \mathrm{~g} / \mathrm{dL}$ para homens.
O risco para o desenvolvimento de lesões por pressão avaliado pela escala de BRADEN foi de $9,6 \pm 0,70$.

Todas as lesões estavam localizadas na região sacral. A avaliação das características das lesões pelo MEASURE estão descritas na Tabela 3.

TABELA 3 - A avaliação das lesões pelos parâmetros do MEASURE e pelo MOWA ${ }^{\circledR}$

\section{Parâmetros}

\begin{tabular}{|c|c|c|c|}
\hline \multirow[b]{2}{*}{ Parâmetros } & \\
\hline & Avaliação Inicial & $\begin{array}{c}1^{\text {a }} \\
\text { Reavaliação }\end{array}$ & $\begin{array}{c}2^{\mathrm{a}} \\
\text { Reavaliação }\end{array}$ \\
\hline Área da ferida ${ }^{1}\left(\right.$ média $\pm D P, \mathrm{~cm}^{2}$ ) & $84,48 \pm 66,58$ & $63,23 \pm 23,48$ & $69,78 \pm 26,71$ \\
\hline \multicolumn{4}{|l|}{ Características Teciduais ${ }^{1}(\%)$} \\
\hline Necrose $^{2}$ & $45,75 \pm 35,70$ & $54,6 \pm 33,64$ & $40,55 \pm 35,10$ \\
\hline Fibrina & $37,93 \pm 26,10$ & $35,7 \pm 33,17$ & $45,2 \pm 27,55$ \\
\hline Granulação & $16,37 \pm 13,88$ & $9,73 \pm 8,81$ & $14,25 \pm 17,88$ \\
\hline Desconhecido & 0 & 0 & 0 \\
\hline \multicolumn{4}{|l|}{ Quantidade do Exudato (\%) } \\
\hline Nenhum & 0 & 0 & 0 \\
\hline Pouco & 70 & 50 & 30 \\
\hline Moderado & 30 & 20 & 10 \\
\hline Grande & 0 & 0 & 0 \\
\hline \multicolumn{4}{|l|}{ Qualidade do Exudato (\%) } \\
\hline Nenhum & 0 & 0 & 0 \\
\hline Seroso & 40 & 30 & 10 \\
\hline Seropurulento & 20 & 0 & 10 \\
\hline Serossanguinolento & 10 & 30 & 20 \\
\hline Sanguinolento & 30 & 10 & 0 \\
\hline \multicolumn{4}{|l|}{ Intensidade da dor (escala analógica) (\%) } \\
\hline 0 & 30 & 30 & 10 \\
\hline 2 & 10 & 10 & 0 \\
\hline 4 & 10 & 30 & 30 \\
\hline 6 & 30 & 0 & 0 \\
\hline 8 & 20 & 0 & 0 \\
\hline \multicolumn{4}{|l|}{ Temporalidade da dor (\%) } \\
\hline Remoção da cobertura & 30 & 30 & 30 \\
\hline Dor contínua & 30 & 0 & 0 \\
\hline Não referiu & 40 & 40 & 10 \\
\hline \multicolumn{4}{|l|}{ Aparência da lesão (\%) } \\
\hline Perda de Epiderme & 30 & 20 & 0 \\
\hline Perda de Subcutâneo & 30 & 10 & 20 \\
\hline Perda cutânea total & 30 & 40 & 20 \\
\hline Ferida completamente necrosada & 10 & 0 & 0 \\
\hline \multicolumn{4}{|l|}{ Descolamento (\%) } \\
\hline Ausente & 100 & 100 & 100 \\
\hline Presente & 0 & 0 & 0 \\
\hline \multicolumn{4}{|l|}{ Tipo de borda (\%) } \\
\hline B2 Delimitada & 50 & 30 & 20 \\
\hline B3 $\quad$ Irregular & 50 & 40 & 20 \\
\hline \multicolumn{4}{|l|}{ Tipo de tecido (\%) } \\
\hline Necrótico & 10 & 0 & 10 \\
\hline Esfacelos+Granulação & 30 & 0 & 30 \\
\hline Esfacelos+Granulação+Necrótico & 10 & 0 & 0 \\
\hline Granulção + Neccrótico & 20 & 40 & 0 \\
\hline Esfacelos+Epitelial+Necrótico & 30 & 10 & 0 \\
\hline Esfacelos + Necrótico & 0 & 20 & 0 \\
\hline \multicolumn{4}{|l|}{ Coloração (\%) } \\
\hline Vermelha & 10 & 0 & 0 \\
\hline Amarela & 70 & 0 & 20 \\
\hline Preta & 10 & 10 & 0 \\
\hline Mista & 10 & 60 & 20 \\
\hline
\end{tabular}

Área da ferida ${ }^{1}$ (média $\pm D P, \mathrm{~cm}^{2}$ )

Características Teciduais ${ }^{1}(\%)$

Quantidade do Exudato (\%)

\section{Qualidade do Exudato (\%)}

Avaliação Inicial

$84,48 \pm 66,58$

$45,75 \pm 35,70$

$37,93 \pm 26,10$

Granulação

Desconhecido

\begin{tabular}{rr}
\hline & Nenhum \\
\hline & Pouco \\
\hline & Moderado \\
\hline Qualidade do Exudato (\%) & Grande \\
\hline & Nenhum \\
\hline & Seroso \\
\hline & Seropurulento \\
\hline & Serossanguinolento \\
\hline Intensidade da dor (escala analógica) (\%) & Sanguinolento \\
\hline
\end{tabular}

Intensidade da dor (escala analógica) $(\%)$

${ }^{1}$ Avaliação da área da lesão e suas características teciduais realizadas pelo programa MOWA.
$16,37 \pm 13,88$

\section{CB}


As figuras 2, 3 e 4 ilustram o processo de cicatrização com a utilização da membrana de $C B$ na fase inicial e após 07 e 15 dias de reavaliação.

Figura 2: Lesão por pressão, paciente 2. Avaliação Inicial (0 dias). Localização Sacral, presença de tecido fibrinoso desvitalizado (seta preta), tecido de granulação escasso (seta amarela).
Figura 3: Lesão por pressão, paciente 2. Primeira reavaliação (7 dias). Localização Sacral, presença de tecido fibrinoso desvitalizado (seta preta), leve melhora no tecido de granulação (seta amarela)

Figura 4: Lesão por pressão, paciente 2. Segunda reavaliação (I5 dias). Localização Sacral, presença de tecido fibrinoso desvitalizado (seta preta), melhora no tecido de granulação (seta amarela).

FIGURA 2 - Lesão por pressão, paciente 2. Avaliação Inicial (0 dias). Localização Sacral, presença de tecido fibrinoso desvitalizado (seta preta), tecido de granulação escasso (seta amarela).

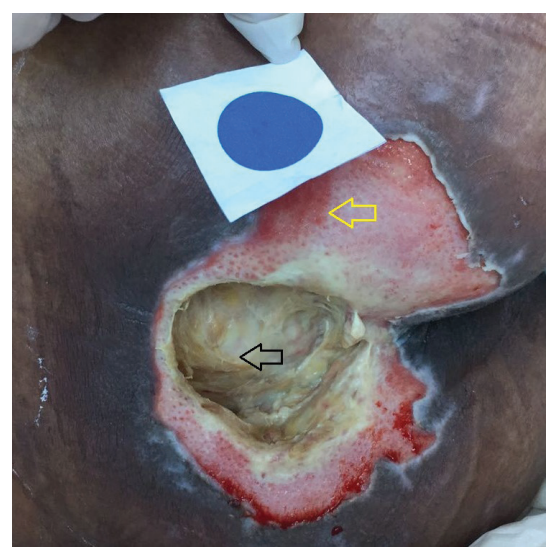

FIGURA 3 - Lesão por pressão, paciente 2. Primeira reavaliação (7 dias). Localização Sacral, presença de tecido fibrinoso desvitalizado (seta preta), leve melhora no tecido de granulação (seta amarela)

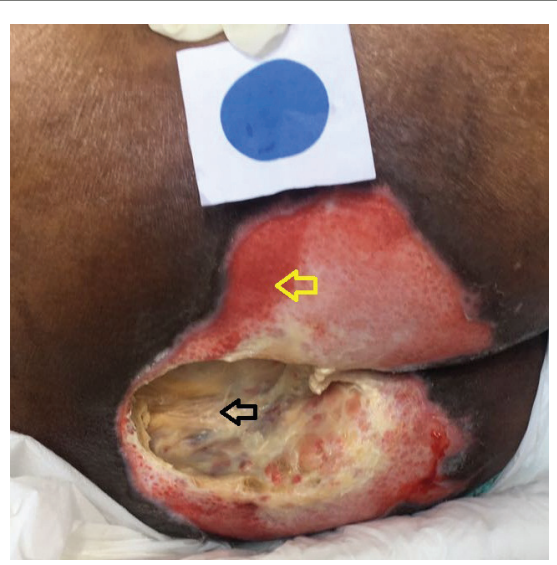

FIGURA 4 - Lesão por pressão, paciente 2. Segunda reavaliação (15 dias). Localização Sacral, presença de tecido fibrinoso desvitalizado (seta preta), melhora no tecido de granulação (seta amarela). 


\section{DISCUSSÃO}

A avaliação do perfil sociodemográfico demonstrou que o sexo feminino foi o que obteve maior predominância no estudo, cerca de $60 \%$. Diferindo da literatura, que aponta que $58 \%$ dos pacientes internados na unidade de terapia intensiva de um hospital universitário eram do sexo masculino e $42 \%$ do sexo feminino ${ }^{15}$. Porém, isto pode estar relacionado ao número reduzido da amostra.

Outra característica avaliada neste estudo foi o Índice de Massa Corporal (IMC), onde a maioria dos pacientes apresentava sobrepeso, segundo a classificação da Organização Mundial de Saúde (OMS). Pode haver implicação do IMC no processo de cicatrização, onde altos índices podem retardar o processo cicatricial, conforme o Manual de Condutas para Úlceras Neurotróficas e Traumáticas (2002) ${ }^{16}$.

Contudo, a média de idade de 60 anos, manteve-se de acordo com a literatura. $\mathrm{O}$ aumento da idade é um fator de risco para o avanço da LP e isto ocorre pelas alterações da pele e do tecido subcutâneo, diminuição da espessura dérmica, do colágeno e atrofia muscular, evidenciando as proeminências ósseas ${ }^{17}$.

As principais comorbidades encontradas nos pacientes deste estudo foram DM (30\%), HAS (20\%) DM e HAS associadas (30\%). Este achado corrobora com o estudo retrospectivo, em que foram utilizados 222 prontuários de pacientes de diferentes patologias, submetidos às cirurgias com mais de duas horas, onde os autores relacionaram o surgimento da LP à diminuição da perfusão tecidual decorrente da hipertensão e diabetes ${ }^{18}$.

Vários são os fatores relacionados ao surgimento das LP em pacientes críticos. Destacam-se a pressão extrínseca associada à faixa etária avançada, o déficit nutricional, a umidade, a imobilidade no leito, a perfusão tecidual diminuída, o uso de drogas vasoativas, a sedação e as comorbidades como diabetes mellitus e doença vascular ${ }^{19}$.

Os níveis séricos de hematócrito $(\mathrm{Ht})$ e hemoglobina $(\mathrm{Hb})$ foram registrados e avaliados, apresentando valores abaixo da taxa esperada, esse achado impacta diretamente na cicatrização da ferida, como é descrito no estudo que enfatiza a diminuição de oxigenação molecular nos casos de anemia, e que no contexto de cicatrização, este fato contribui para retardo do processo, visto a carência em atender as demandas do tecido lesado ${ }^{20}$.
A UTI, de modo geral, é um ambiente hostil, predisponente ao aparecimento das LP.Apesar de ser o local indicado para o tratamento de pacientes críticos, é também classificada como um dos ambientes hospitalares mais agressivos, tensos e traumatizantes ${ }^{17}$.

O escore médio encontrado pela escala de Braden foi de $9,6 \pm 0,70$, onde o maior escore foi 10 e o menor escore foi 8 , que correspondem a um risco elevado e muito elevado dos pacientes desenvolverem lesões por pressão. Esses achados corroboram com o estudo onde observou-se um escore de risco elevado $(<\mathrm{II})$ pela escala de Braden em I I clientes com LP em Unidade de Terapia Intensiva adulto de um hospital público no Rio de Janeiro ${ }^{21}$.

Todas as lesões por pressão estavam localizadas na região sacral, esta predominância anatômica também está descrita em outros estudos ${ }^{17,22}$. Estão sumarizados na tabela 4 estudos que corroboram com a predominância sacral.

A avaliação das características foi realizada pelo protocolo MEASURE, destinado para avaliação de feridas, executado comumente na rotina hospitalar e ambulatorial. Este protocolo é utilizado para em estudos de lesões cutâneas ${ }^{14}$. Quanto a cicatrização da ferida, foi observada redução entre a área inicial e a área final das lesões $\left(-14,7 \mathrm{~cm}^{2}\right)$.

As características teciduais foram avaliadas pelo programa MOWA® (MobileWound Analyzer $®$ ) cujas vantagens são: fácil utilização, registro claro e permanente, evita o contato com a pele, permite a avaliação da planimetria, cálculo preciso da área da ferida, fornece o tratamento, permite telemedicina.

Quanto às características teciduais avaliadas pelo MOWA®, foi verificado um aumento do tecido de granulação na terceira avaliação dos pacientes, quando comparada com a segunda avaliação de $9,73 \%$ para $14,25 \%$, respectivamente. Este achado nos sugere que CB, comportou-se como um indutor de tecido de granulação, como é sugerido no estudo das células mesenquimais do biopolímero, esse fenômeno tem fundamental importância na diminuição da profundidade das feridas cutâneas ${ }^{23}$.

Em um estudo randomizado observou-se que as úlceras foram mais superficiais no final do período de observação ( 120 dias) em mais de $80 \%$ dos pacientes com úlceras venosas que receberam o curativo de $B C$ do que no grupo controle $(60 \%)$. Isso pode indicar, segundo os

TABELA 4 - Estudos que corroboram com predominância sacral

\begin{tabular}{l|c|c}
\hline Autor (data) & Região & $\%$ \\
\hline & Sacral & $47 \%$ \\
Borghardt (2015) & Trocantérica & $19 \%$ \\
& Maleolar & $16 \%$ \\
\hline & Sacrococcígea & $60,7 \%$ \\
Sousa (2006) & Glúteo & $28,6 \%$ \\
\hline
\end{tabular}


autores, que os curativos BC atuaram como indutor de remodelação tecidual ${ }^{6}$.

É importante destacar que não houve sinais de reações tóxicas associadas ao biopolímero, mostrando que esta é uma membrana atóxica e biocompatível ${ }^{8}$. E apresentou características essenciais como curativo ideal, sendo esta uma possível opção de cobertura.

Os resultados obtidos nesta pesquisa, apesar de preliminares, trazem incentivo para a continuidade do estudo e possíveis inovações no ramo dos biomateriais.

\section{CONCLUSÃO}

A membrana de celulose bacteriana foi eficaz para o tratamento lesões por pressão, já que se comportou como indutor do tecido de granulação, diminuindo a profundidade da lesão. Sendo um curativo inovador, de baixo custo e importante alternativa terapêutica frente à problemática.

Muitos fatores influenciam no sucesso do processo cicatricial, como: idade, doenças crônicas, estado nutricional, problemas cardiovasculares, restrição ao leito, e ainda impactam neste processo as forças de fricção, cisalhamento e pressão. A população deste estudo, oriunda do setor de terapia intensiva, é afetada por alguns destes fatores, que interferem no processo de cicatrização da ferida. Deste modo, torna-se mais delicada a problemática vivenciada. Porém, a visão holística sobre estes pacientes e a atuação multiprofissional podem otimizar e viabilizar este processo com maiores chances de sucesso. 


\section{REFERÊNCIAS}

I. NPUAP (National Pressure Ulcer Advisory Panel). NPUAP Pressure Injury Stages. Press Inj Stages [Internet]. 2016;all. Available from: http://www.npuap.org/resources/educational -and-clinical-resources/npuap-pressure-injury-stages/

2. Campos SF, Chagas ÂCP, Costa ABP, França REM, Jansen AK. Fatores associados ao desenvolvimento de úlceras de pressão: o impacto da nutrição. Rev Nutr [Internet]. 2010;23(5):70314. Available from: http://www.scielo.br/scielo.php?script=sci_arttext\&pid=SI 4 I5-527320 $0000500002 \& \operatorname{lng}=$ pt\&tlng=pt

3. Santos CT, Oliveira MC, Pereira AGDS, Suzuki LM, Lucena ADF. Indicador de qualidade assistencial úlcera por pressão: análise de prontuário e de notificação de incidente. Rev Gauch Enferm [Internet]. 20।3;34(I): I I I-8. Available from: http://www.scielo.br/scielo.php?script=sci_arttext\&amp\%5Cnpid $=$ S $1983-|44720| 3000|000| 4$

4. Borghardt AT, Prado TN, Araújo TM, Rogenski NMB, Bringuente MEO. Evaluation of the pressure ulcers risk scales with critically ill patients: a prospective cohort study. Rev Lat Am Enfermagem [Internet]. 2015;23(I):28-35. Available from: http://www.scielo.br/scielo.php?script=sci_arttext\&pid=SO I 04- | | 6920 I 5000 I 00028\&lng=en\&tlng=en

5. Recouvreux. Desenvolvimento de Novos Biomateriais Baseados em Celulose Bacteriana para Aplicações Biomédicas e de Engenharia de Tecidos. Tese doutorado em Eng Quim Univ Fed St Catarina. 2008;Único: I 45.

6. Cavalcanti LM, Pinto FCM, Oliveira GM, Lima SVC, Aguiar JLDA, Lins EM. Efficacy of bacterial cellulose membrane for the treatment of lower limbs chronic varicose ulcers: a randomized and controlled trial. Rev Col Bras Cir [Internet]. 20I7;44(I):72-80. Available from: http://www.scielo.br/scielo.php?script=sci_arttext\&pi$d=S 0100-699|2017000100072 \&| n g=e n \& t \mid n g=e n$

7. Pinto FCM, De-Oliveira ACAX, De-Carvalho RR, GomesCarneiro MR, Coelho DR, Lima SVC, et al.Acute toxicity, cytotoxicity, genotoxicity and antigenotoxic effects of a cellulosic exopolysaccharide obtained from sugarcane molasses. Carbohydr Polym. 2016;137:556-60.

8. Castro CMMB, Aguiar JLA, Melo FAD, Silva WTF, Marques E, Silva DB. Citotoxicidade do biopolímero de cana-de-açúcar. AnFacMedUnivFedPernamb. 2004;49(2): I I 9-23.

9. Lima SVC, Rangel AEO, Lira MMM, Pinto FCM, Campos Júnior O, Sampaio FJB, et al. The Biocompatibility of a Cellulose Exopolysaccharide Implant in the Rabbit Bladder When Compared With Dextranomer Microspheres Plus Hyaluronic Acid. Urology. 20I5;85(6).

10. Pita PCC, Pinto FCM, Lira MMM, Melo FAD, Ferreira LM, et al. Biocompatibility of the bacterial cellulose hydrogel in subcutaneous tissue of rabbits I. Acta Crúrgica Bras. 20I5;30(4):296-300.
I I. Oliveira MAP,Velarde GC, Moreira De Sá RA. Entendendo a pesquisa clínica $V$ : relatos e séries de casos. Femina [Internet]. 20 I5;43(5):235-8.Available from: http://files.bvs.br/ upload/S/0I00-7254/20I5/v43n5/a5320.pdf

12. Oliveira TMV. Amostragem não Probabilística: Adequação de Situações para uso e Limitações de amostras por Conveniência, Julgamento e Quotas. Adm Line [Internet]. 200 I;2(3): I-7. Available from: https://goo.gl/skzASC

13. Keast DH, Bowering CK, Evans AW, Mackean GL, Burrows C, D'Souza L. MEASURE:A proposed assessment framework for developing best practice recommendations for wound assessment.Wound Repair Regen. 2003;12(3 Suppl):SI-I7.

14. Crozeta K.Avaliação clínica e epidemiológica das úlceras por pressão em um hospital de ensino. 2009;90. Available from: http://www.ppgenf.ufpr.br/DissertaçãoKarlaCrozeta.pdf

I5. Favarin SS, Camponogara S. Perfil dos pacientes internados na unidade de terapia intensiva adulto de um hospital universitário. Rev Enferm da UFSM [Internet]. 20I2;2(2):3209. Available from: http://cascavel.ufsm.br/revistas/ojs-2.2.2/ index.php/reufsm/article/view/5 I78

16. BRASIL $M$ da SS de P de SD de AB. Manual de Condutas para Úlceras Neurotróficas e Traumáticas [Internet]. Cadernos de Reabilitação em Hanseníase. 2002. I.55. Available from: http://bvsms.saude.gov.br/bvs/publicacoes/manual_feridas_final.pdf

17. Fernandes NCS, Torres GV,Vieira D. Fatores de risco e condições predisponentes para úlcera de pressão em pacientes de terapia intensiva. Rev Eletrônica Enferm. 2008; 10:733-46.

18. Lumbley JL, Ali SA, Tchokouani LS. Retrospective review of predisposing factors for intraoperative pressure ulcer development. J Clin Anesth. 2014;26(5):368-74.

19. Sewchuk D, Padula C, Osborne E. Prevention and Early Detection of Pressure Ulcers in Patients Undergoing Cardiac Surgery. AORN J. 2006;84(I).

20. Afonseca MA, Almeida RR, Reis SRA, Medrado ARAP. Repercussão de doenças sistêmicas no reparo tecidual. Rev Bahiana de Odontologia. 2012;3(I):63-75

21. Sousa CA, Santos I, Silva LD. Aplicando recomendações da Escala de Braden e prevenindo úlceras por pressão: evidências do cuidar em enfermagem. Rev Bras Enferm [Internet]. 2006;59(3):279-84. Available from: http://www.scielo.br/scielo.php?script=sci_arttext\&pi$d=$ S0034-7 I 672006000300006\&lng=pt\&nrm=iso\&tlng=pt

22. Silva DRA, Bezerra SMG, Costa JP, Luz MHBA, Lopes VCA, Nogueira LT. Curativos de lesões por pressão em pacientes críticos: análise de custos.2017;5-12.

23. Fragoso AS, Silva MB, Melo CP, Aguiar JLA, Rodrigues CG, Medeiros PL, et al. Dielectric study of the adhesion of mesenchymal stem cells from human umbilical cord on a sugarcane biopolymer.J Mater Sci Mater Med. 20 I4;25(I):229-37. 